Journal for ImmunoTherapy of Cancer

\title{
Quality of life in long-term survivors of advanced melanoma treated with checkpoint inhibitors
}

\author{
Maha Mamoor, ${ }^{1}$ Michael A Postow, ${ }^{2}$ Jessica A Lavery, ${ }^{2}$ Shrujal S Baxi, ${ }^{3}$ \\ Niloufer Khan, ${ }^{2}$ Jun J Mao, ${ }^{2}$ Lauren J Rogak, ${ }^{2}$ Robert Sidlow, ${ }^{2}$ Bridgette Thom, ${ }^{2}$ \\ Jedd A Wolchok, ${ }^{2}$ Deborah Korenstein (D) ${ }^{2}$
}

To cite: Mamoor M, Postow MA, Lavery JA, et al. Quality of life in long-term survivors of advanced melanoma treated with checkpoint inhibitors. Journal for ImmunoTherapy of Cancer 2020;8:e000260. doi:10.1136/ jitc-2019-000260

Accepted 05 February 2020

Check for updates

(C) Author(s) (or their employer(s)) 2020. Re-use permitted under CC BY-NC. No commercial re-use. See rights and permissions. Published by BMJ.

${ }^{1}$ Health Outcomes Group, Memorial Sloan Kettering Cancer Center, New York, New York, USA

${ }^{2}$ Medicine, Memorial Sloan Kettering Cancer Center, New York, New York, USA

${ }^{3}$ Flatiron Health Inc, New York, New York, USA

Correspondence to Dr Deborah Korenstein; korenstd@mskcc.org

\section{ABSTRACT}

Background Immune checkpoint inhibitors (Cls) have revolutionized treatment of advanced melanoma, leading to an emerging population of long-term survivors.

Survivors' quality of life (QOL) and symptom burden are poorly understood. We set out to evaluate symptom burden and QOL in patients with advanced melanoma alive more than 1 year after initiating $\mathrm{Cl}$ therapy.

Methods Cross-sectional surveys, accompanied by chart review of patients with advanced melanoma treated with Cls at Memorial Sloan Kettering Cancer Center, completed therapy, and were alive $>1$ year after treatment initiation. Surveys were administered between February and August 2018. Surveys included: European Organization for Research and Treatment of Cancer Quality of Life Questionnaire-Core 30, EuroQ0L, items from PatientReported Outcomes version of the Common Terminology Criteria for Adverse Events and Fatigue Severity Scale. Results We included 90 patients. The most common $\mathrm{Cl}$ regimens were ipilimumab plus nivolumab $(53 \%)$ and pembrolizumab (41\%); most patients $(71 \%)$ were not treated in clinical trials. Median time from $\mathrm{Cl}$ therapy initiation was 40 months and from last dose was 28 months. Fatigue was reported by $28 \%$, with higher fatigue scores in women than men; $12 \%$ reported difficulty sleeping. Aching joints (17\%) and muscles (12\%) were fairly common. Level of functioning was generally high Overall QOL was excellent though $40 \%$ reported 'some or moderate' problems with anxiety/depression and $31 \%$ with pain/discomfort.

Conclusions After $\mathrm{Cl}$ therapy, long-surviving advanced melanoma patients commonly report fatigue but otherwise have moderate symptom burden and good QOL. Ensuring appropriate symptom management will optimize clinical outcomes for these patients.

\section{INTRODUCTION}

Immune checkpoint inhibitors (CIs), in particular inhibitors of the cytotoxic T-lymphocyteassociated antigen 4 and programmed death 1 (PD-1), have revolutionized the treatment of advanced melanoma. Ipilimumab, nivolumab and pembrolizumab, alone or in combination (ipilimumab plus nivolumab) have led to dramatic improvements in survival. In some patients, CI therapy may lead to long-term survival, ${ }^{1}$ resulting in the emergence of a new population of cancer survivors. ${ }^{2}$

While some clinical trials have followed patients for as long as 5 years, most quality of life (QoL) evidence on CIs in melanoma is limited to 1 or 2 years follow-up time. Despite the common occurrence of immune-related adverse events (irAEs), good QoL is generally maintained in melanoma patients in CI clinical trials. ${ }^{3}$ However, patients treated outside of trials may have higher rates of adverse events and lower QoL. ${ }^{4}$ The clinical experiences and needs of long-term melanoma survivors treated with CI, including symptom burden, QoL and health services needs, are not well described. ${ }^{5}$ Such information is critical for optimizing care for this growing survivor group. Memorial Sloan Kettering Cancer Center (MSK) provides care to many long-term melanoma survivors who have been treated with CIs. We performed a crosssectional study, accompanied by chart review, to measure symptom burden and long-term QoL in this cohort of patients.

\section{METHODS}

\section{Study design}

We used an institutional database to identify potential study participants in two ways: by identifying participants in two CIs clinical trial expanded access programs (Expanded Access Program for Pembrolizumab and Expanded Access Program for Nivolumab in Combination with Ipilimumab) and by generating a list of potentially eligible patients treated in routine clinical practice off-protocol. Eligible patients were those with unresectable stage III or IV melanoma treated with CI at MSK who were alive at least 12 months after initiation of CI therapy. We included those able to speak and read English, able to provide informed consent, 
diagnosed with melanoma at age $\geq 18$ years, treated with either single agent or combination CI, and who had received no other systemic therapy after initiation of CI (interval radiation or surgery was permitted). We excluded patients identified by the care team as having acute symptomatic progression or being poor study candidates due to interpersonal or psychosocial factors. Once patients were enrolled, we did not exclude those with previously unappreciated progression.

We checked eligibility through medical record review of all patients enrolled in the two expanded access protocols and a randomly selected group of 200 routine practice patients, reviewing charts and contacting patients until reaching our accrual goal of 100 patients. Given that the study was exploratory, we did not perform a power calculation but believed it was feasible to enroll 100 participants. Patients identified as eligible were contacted via a mailed letter, email, and/or phone, and were given the option of completion online, over the phone with a researcher or face to face. Study data were collected and managed using Research Electronic Data Capture electronic data capture tools. ${ }^{67}$ All patients were enrolled between February and August 2018.

\section{Study measures}

The survey consisted of five instruments and openended questions related to symptoms and problems: European Organization for Research and Treatment of Cancer Quality of Life Questionnaire-Core 30 (EORTC QLQ-C30) V.3.0, EuroQOL (EQ-5D-3L), items from the Patient-Reported Outcomes version of the Common Terminology Criteria for Adverse Events (PRO-CTCAE), Fatigue Severity Scale (FSS) and the Comprehensive Score for Financial Toxicity ${ }^{8-12}$ (to be presented separately) (online supplementary figure 1 ). Specific symptoms were measured using the EORTC QLQ-C30, a 30-item questionnaire that has been used extensively to assess healthrelated QoL in patients with cancer. The EQ-5D-3L is a non-cancer-related QoL measurement tool that includes five dimensions (mobility, self-care, activity, pain and anxiety/depression) assessed on three levels (eg, no problems, moderate problems, extreme problems). The PRO-CTCAE is a patient-reported outcome measure that includes 124 items representing 78 symptomatic toxicities adapted from the CTCAE. PRO-CTCAE assesses symptom presence and impact; specific items were chosen based on potential irAE. The FSS questionnaire is a nine-item unidimensional scale to assess the impact of fatigue on daily life.

We performed chart abstraction for all participants to assess cancer stage, Eastern Cooperative Oncology Group (ECOG) status, Charlson Comorbidity Index and medical conditions at the time of $\mathrm{CI}$ initiation, organ-specific irAE during or after CI treatment, and number of surgeries after CI treatment. Chart abstractions were performed by MM, BT, RS, NK and DK, with questions resolved through group discussion.

\section{Statistical analyses}

The primary study aim was to describe global and specific elements of symptom burden and QoL in patients who were alive at least 12 months from the original dose of CI with sustained tumor control. Since symptoms may differ between patients on and off therapy, we limited analyses to patients who were no longer receiving CI at the time of the survey. Patients were considered off therapy if clinical notes prior to survey completion indicated no plans for continued therapy. We used descriptive statistics to summarize demographics, cancer characteristics and CI regimen. Categorical and continuous survey responses were summarized by percentages and means, respectively. Patients were stratified based on time since completion of CI therapy ( $<24$ months vs 24 to $<36$ months vs $36+$ months). For the EORTC, we collapsed not at all/very little and quite a bit/very much. For the PRO-CTCAE, we collapsed none/mild (1-2), moderate (3) and severe/ very severe (4-5). For the FSS, we collapsed disagree (1-3), neutral (4) and agree (5-7). The FSS score was calculated by taking a sum across all 9 items of the instrument (range: 0-63). For the EQ-5D-3L, we collapsed some/moderate and yes and calculated the global health status score by substracting 1 from the raw score, dividing this by the range and multiplying by 100 . We used $\mathrm{X}^{2}$ and Fisher's exact tests to analyze associations of survey responses with sex and time off treatment,sex and toxicities, and between the EQ-5D-3L score and receipt of combination therapy. We applied the Kruskal-Wallis test to analyze the association between global health score and time from treatment, the EQ-5D-3L domains and time from treatment, and FSS score and time from treatment, and FSS score and receipt of combination therapy, and the Wilcoxon rank-sum test to analyze the association between FSS score and sex.

As a secondary aim, we sought to understand healthcare utilization patterns. Using open-ended questions, we asked participants to list the providers they saw outside of MSK in the last year. Analyses were performed using Stata (V.15.0) and SAS V.9.4. ${ }^{13}$

\section{RESULTS}

We approached 274 eligible patients; 107 (39\%) responded and agreed to participate. Non-responders were of similar age as participants (mean 63 vs 65 years) and were slightly more likely to be male ( $65 \%$ vs $57 \%$ ). We were unable to collect other information about nonresponders. One hundred and six patients completed the survey. Of those, 16 remained on CI treatment and were excluded, leaving 90 off-treatment participants for analysis.

Demographic characteristics are shown in table 1. Nearly all (93\%) patients were white; $43 \%$ were female. The median age at time of survey was 65 years (IQR 54-74 years). All patients had a Charlson comorbidity index of 0 at treatment initiation and very few (7 (8\%)) had pre-existing autoimmune illnesses (data not shown). 
Table 1 Patient demographics

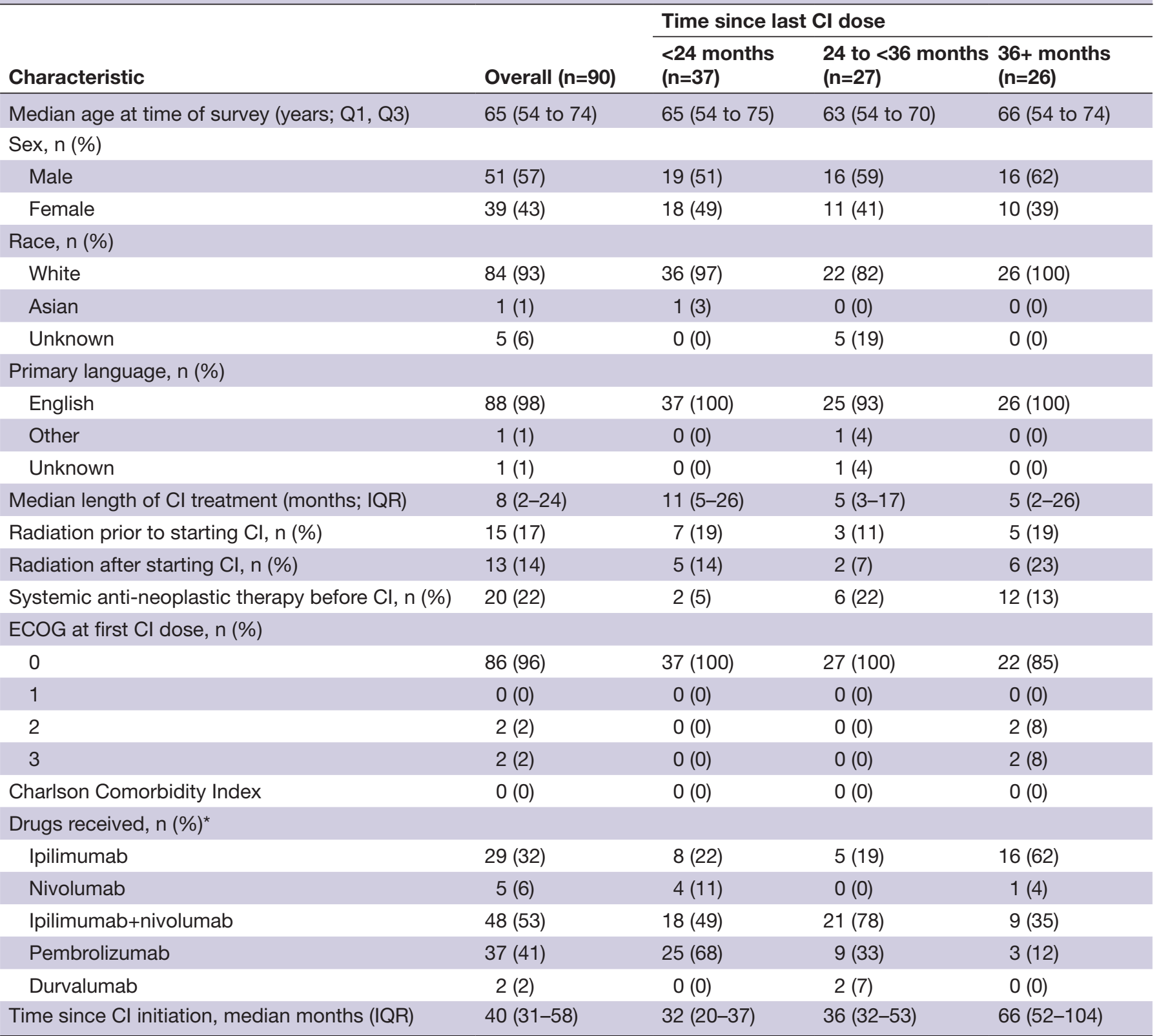

*Individual patients may have received more than one drug, or a drug more than once in different regimens, therefore, totals exceed the total number of patients.

$\mathrm{Cl}$, checkpoint inhibitor.

Twenty-two per cent had received systemic antineoplastic therapy prior to CI; $12(17 \%)$ had been treated with radiation therapy (RT) prior to CI and 13 (14\%) received RT after $\mathrm{CI}$ initiation.

The most commonly received CI regimen was ipilimumab plus nivolumab (53\%), followed by pembrolizumab $(41 \%)$. Median length of CI treatment was 8 months (IQR: 2-24 months). Most participants $(\mathrm{n}=64,71 \%)$ were not treated with CI within a clinical trial; $15(17 \%)$ were treated only on a clinical trial protocol and $11(12 \%)$ received CI both on and off protocol. The median time from initiation of CI therapy to survey completion was 40 months (IQR: 31-58 months) and the median time from the last dose of CI therapy to survey completion was 28 months (IQR: 17-40 months), with 15 patients (17\%) off treatment $<12$ months, 22 (24\%) from 12 to $<24$ months, $27(30 \%)$ from 24 to $<36$ months, $7(7.8 \%)$ from 36 to $<48$ months and $19(21 \%) \geq 48$ months.

Table 2 shows all domains of PRO-CTCAE by time since last CI dose. The most commonly reported symptoms on the PRO-CTCAE were aching joints (17\%), aching muscles $(12 \%)$ and headache $(6 \%)$, with no meaningful differences in frequency or severity based on time since treatment or receipt of combination versus single-agent therapy. PRO-CTCAE results also did not differ based on sex (table 3). Rates of difficulty with physical, role (eg, 


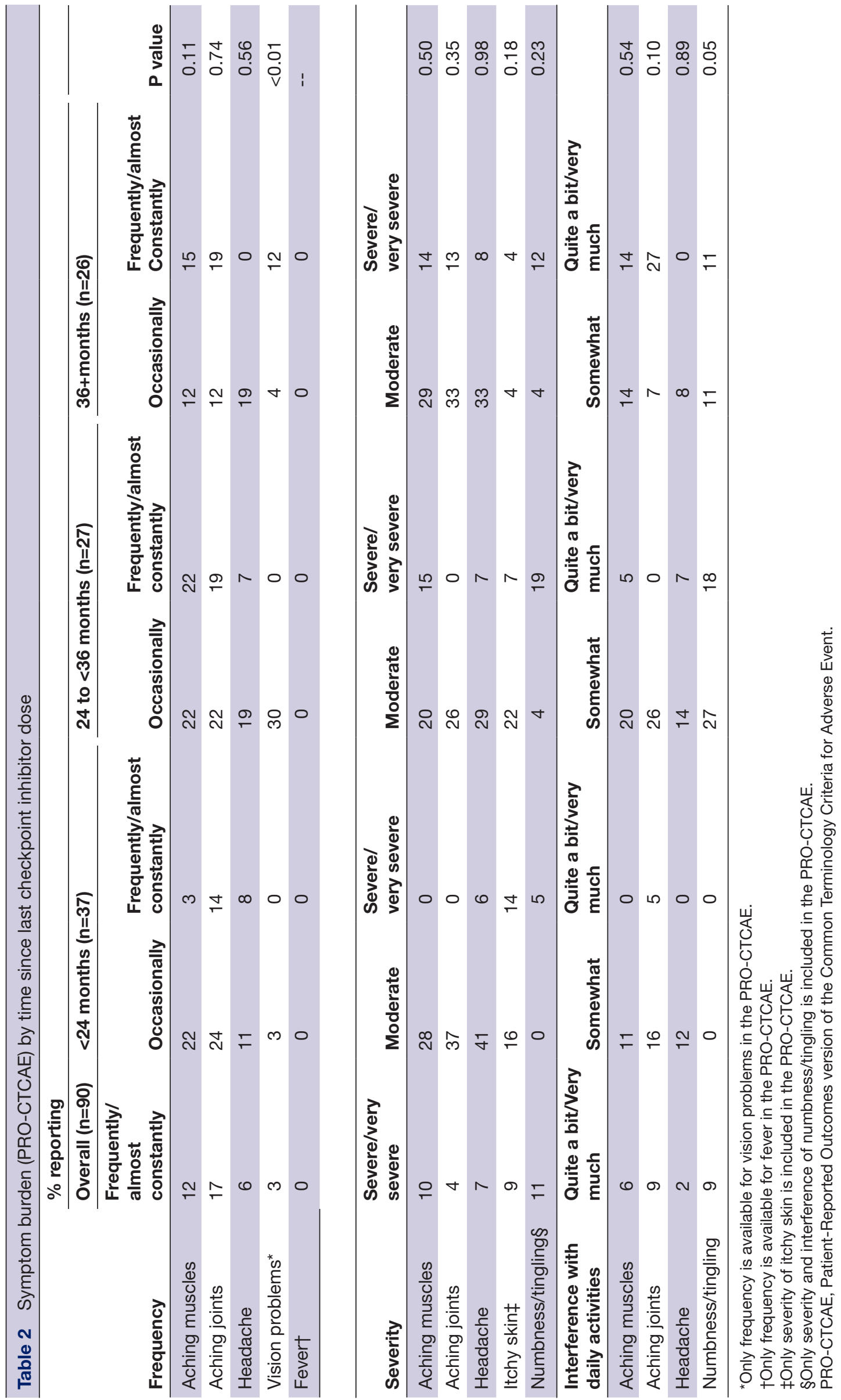

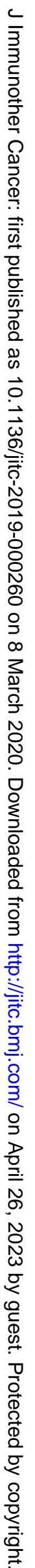


Table 3 Symptom burden (PRO-CTCAE) by sex

\begin{tabular}{|c|c|c|c|c|}
\hline & \multicolumn{4}{|c|}{$\%$ Reporting* } \\
\hline & $\begin{array}{l}\text { Overall } \\
n=90\end{array}$ & $\begin{array}{l}\text { Male } \\
\mathrm{n}=51\end{array}$ & $\begin{array}{l}\text { Female } \\
\mathrm{n}=39\end{array}$ & $P$ value \\
\hline \multicolumn{5}{|l|}{ Frequency } \\
\hline Aching muscles & 12 & 12 & 13 & 0.68 \\
\hline Aching joints & 17 & 18 & 15 & 0.09 \\
\hline Headache & 6 & 2 & 10 & 0.28 \\
\hline Vision problems $\dagger$ & 3 & 4 & 3 & $>0.95$ \\
\hline Fever $\ddagger$ & 0 & 0 & 0 & -- \\
\hline \multicolumn{5}{|l|}{ Severity } \\
\hline Aching muscles & 10 & 8 & 11 & 0.91 \\
\hline Aching joints & 4 & 4 & 4 & 0.78 \\
\hline Headache & 7 & 0 & 16 & 0.19 \\
\hline Itchy skin§ & 9 & 12 & 5 & 0.26 \\
\hline Numbness/tinglingף & 11 & 12 & 10 & 0.66 \\
\hline \multicolumn{5}{|l|}{ Interference } \\
\hline Aching muscles & 6 & 4 & 7 & 0.22 \\
\hline Aching joints & 9 & 4 & 14 & 0.32 \\
\hline Headache & 2 & 0 & 5 & 0.80 \\
\hline Numbness/tingling & 9 & 10 & 8 & 0.25 \\
\hline
\end{tabular}

*Percentage reporting severe/very severe.

†Only frequency is available for vision problems in the PROCTCAE.

‡Only frequency is available for fever in the PRO-CTCAE. §Only severity of itchy skin is included in the PRO-CTCAE. ПOnly severity and interference of numbness/tingling is included in the PRO-CTCAE.

work around the home), emotional, cognitive or social functioning on the EORTC QLQ-C30 varied (table 4), with more difficulty with physical and work functioning in patients $>3$ years from CI completion.

Fatigue was common. Overall, 28\% of patients reported being easily fatigued on the FSS, $27 \%$ that fatigue interferes with physical functioning, $22 \%$ that fatigue interfered with social functioning and $20 \%$ that fatigue causes frequent problems. The level of fatigue appeared highest in the group who had completed CI 24 to $<36$ months prior, with $26 \%$ in that group agreeing that fatigue causes frequent problems and 37\% agreeing that it interferes with physical functioning (table 5), although acrossgroup differences were not statistically significant. The overall FSS score was 19.5 (IQR: 12-35); FSS scores did not differ based on time since CI therapy $(\mathrm{p}=0.83)$ but were higher (suggesting more fatigue) in females than males (24 vs $18, \mathrm{p}=0.034$ ) and in patients who received combination therapy than in those who received only single-agent (20 vs 19, $\mathrm{p}=0.027$ ). On the EORTC QLQC30, 11 (12\%) patients reported difficulty sleeping; rates ranged from $5 \%$ to $19 \%$ across groups but differences were not significant, and $14 \%$ reported trouble taking a long walk (table 4 ).
Overall QOL was excellent, with a median EQ-5D-3L score of 5.5 (IQR: 5-7) and a median global health score (EORTC QLQ-C30) of 83\%; global health scores did not differ based on time since treatment completion, receipt of combination therapy or sex. On the EORTC QLQC30, $11(12 \%)$ patients reported difficulty sleeping; rates ranged from $5 \%$ to $19 \%$ across groups but differences were not significant, and $14 \%$ reported trouble taking a long walk (table 4). While few patients responded 'yes' on the EQ-5D-3L to having problems in any dimension, many reported 'some or moderate' problems with usual activities (23\%), anxiety/depression (40\%) and pain/ discomfort $(31 \%)$; these rates did not differ based on time since CI treatment or sex (table 6). Moderate problems with mobility and pain on the EQ-5D-3L appeared more prevalent in patients surviving longer since CI completion, (table 6), though differences were not statistically significant.

Most patients $(\mathrm{n}=77(86 \%))$ had seen a healthcare provider outside of MSK in the 12 months prior to the study, including a therapist (eg, mental, physical, speech) $(\mathrm{n}=14(16 \%))$, a nurse practitioner, physician assistant or certified registered nurse practitioner $(6(7 \%))$ or a dentist (2 (2\%)). Physicians outside MSK seen by participants included primary care physicians (PCPs) (37 $(41 \%))$, medical subspecialists $(30(33 \%))$, surgeons (21 $(23 \%))$, dermatologists (20 (22\%)), ophthalmologists $(12(13 \%))$, oncologists $(12(13 \%))$ and gynecologists $(7$ $(8 \%))$.

The most common irAEs at any time since CI initiation were rash $(38 \%)$, colitis (27\%), hypothyroidism (20\%), hepatitis $(17 \%)$ and hypophysitis $(11 \%)$ (table 7$)$. Musculoskeletal toxicities were more common among women compared with men ( $13 \%$ vs $2 \%)$; prevalences of other irAEs were similar in men and women; small numbers of patients with specific irAEs precluded performing formal statistical testing. Notably, patients with endocrine irAEs were more likely to report fatigue compared with those who did not experience endocrine problems $(46 \%$ vs $21 \%$.)

\section{DISCUSSION}

We found high rates of fatigue but otherwise moderate symptom burden and good QoL in patients with advanced melanoma currently off therapy and surviving at least 1 year after CI therapy initiation, despite a relatively high prevalence of having ever experienced an irAE. In the PRO-CTCAE, headache, aching joints and aching muscles were the most commonly reported symptoms in terms of both frequency and severity. Few patients reported definitive mental health problems like anxiety or depression, though many responses indicated 'some' or 'moderate' symptoms, and rates of sleep difficulties were overall similar to those in the general population. ${ }^{14}$

Notably, $28 \%$ of patients in our study reported being easily fatigued and $20 \%$ reported fatigue causing frequent problems. Our results are consistent with robust evidence 


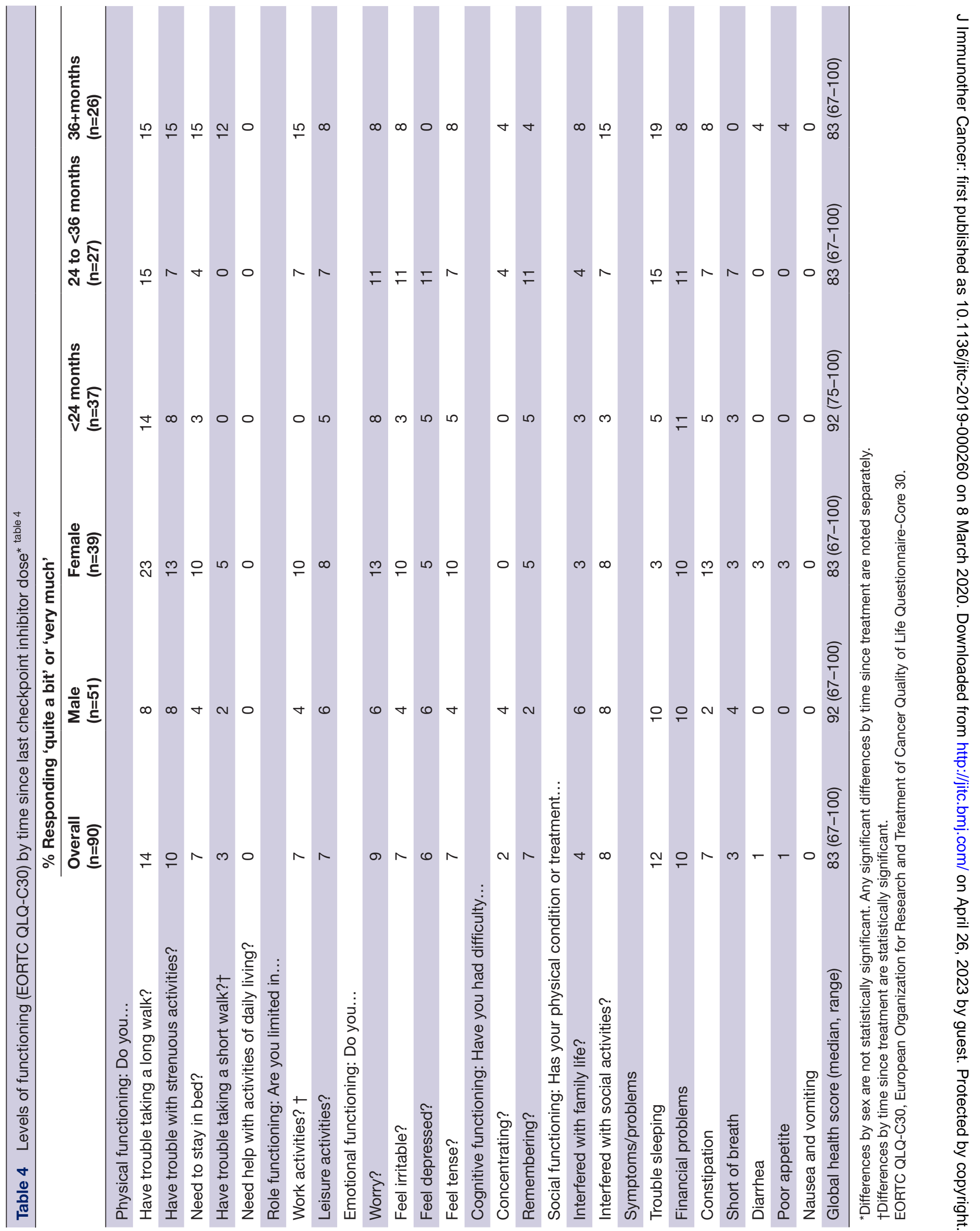




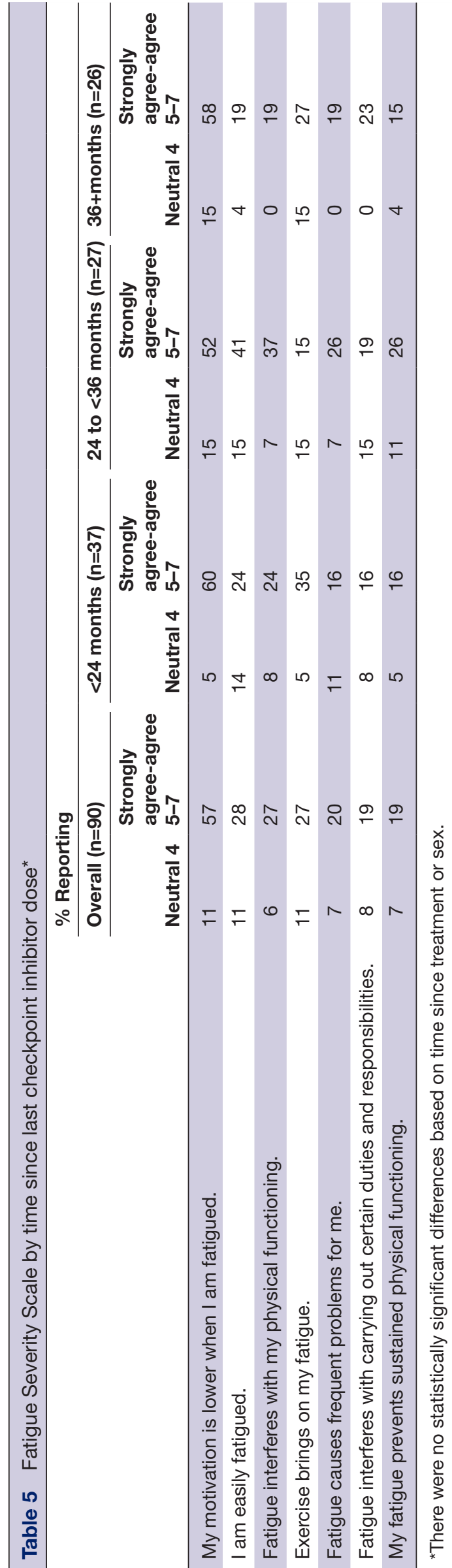

that fatigue is a common problem across populations of cancer survivors with prevalence as high as $40 \%$ and is the most common adverse event in patients on CIs. ${ }^{15-19}$ Findings regarding fatigue in melanoma survivors are mixed. A study of survivors with metastatic melanoma found very high rates of fatigue $(90 \%)$, but most patients were receiving ongoing treatment with CIs or BRAF-MEK inhibitors. ${ }^{20}$ A large study of long-term melanoma survivors found rates of fatigue that were comparable to the general population, ${ }^{21}$ but most had early stage disease. Our study is unique in evaluating long-term survivors of advanced melanoma, previously treated with CI and currently off treatment, including 53 patients surviving $>2$ years at the time of the survey; it reflects a population that is likely growing. Further, the higher rate of fatigue in women than men in our sample is notable. Findings regarding gender differences in fatigue in cancer survivors have been mixed, ${ }^{22-24}$ though some studies have found higher rates of fatigue in women ${ }^{22}$; such differences should be explored in future studies. Our findings of slightly higher fatigue scores in patients who had received combination therapy and that patients with endocrine irAEs may be more likely to be fatigued are interesting and important to address further in the future. As both oncologists and others care for the growing group of long-term cancer survivors treated with CIs, awareness about fatigue and its treatment is crucial. ${ }^{25}$

Our findings regarding QoL are reassuring and are consistent with clinical trials showing that patients on CI maintain QoL better than those treated with other agents. ${ }^{3} 2627$ Our study, however, includes survivors no longer receiving treatment, reflects longer-term symptom burden and captures the experiences of patients treated both within and outside of clinical trials. One small study of long-term melanoma survivors treated with ipilimumab enrolled a similar population to ours and found generally excellent performance status after more than 2 years. ${ }^{2}$ Our study adds to this literature by assessing QoL and including patients treated with PD-1 and programmed death ligand 1 (PD-L1) in combination with ipilimumab, over half of whom were alive more than 2 years after CI completion.

A 2015 study of QoL in long-term melanoma survivors found poorer QoL and higher symptom burden than our study, ${ }^{28}$ but many of those patients were presumably treated before the widespread use of PD-1-based immunotherapy and may not be comparable. Populationbased studies have found that long-term melanoma survivors had similar QoL compared with non-cancer controls ${ }^{2930}$ but it is likely that few in these cohorts had advanced disease. Comparisons with other long-term survivor groups are challenging, since there are few similar populations of patients with advanced disease and durable survival. Such populations might include survivors of lymphoma, in whom findings regarding QoL are mixed, ${ }^{31}$ and breast cancer, whose overall QoL appears comparable to controls but who may report impediments in functioning. ${ }^{32}$ In our study, global QoL exceeded that 
Table 6 Quality of Life (EQ-5D-3L) by time since last checkpoint inhibitor dose* $\%$ Reporting symptoms†

\begin{tabular}{|c|c|c|c|c|c|c|c|c|}
\hline & \multicolumn{2}{|l|}{ Overall $(n=90)$} & \multicolumn{2}{|c|}{$<24$ months $(n=37)$} & \multicolumn{2}{|c|}{24 to $<36$ months $(n=27)$} & \multicolumn{2}{|l|}{$\begin{array}{l}36+\text { months } \\
(\mathrm{n}=26)\end{array}$} \\
\hline & Some/ moderate & Yes & Some/moderate & Yes & Some/moderate & Yes & Some/moderate & Yes \\
\hline Mobility & 19 & 0 & 14 & 0 & 19 & 0 & 27 & 0 \\
\hline Self-care & 4 & 0 & 5 & 0 & 0 & 0 & 8 & 0 \\
\hline Activity & 23 & 0 & 24 & 0 & 22 & 0 & 23 & 0 \\
\hline Pain & 30 & 1 & 22 & 3 & 33 & 0 & 39 & 0 \\
\hline Anxiety & 39 & 1 & 38 & 0 & 37 & 4 & 42 & 0 \\
\hline
\end{tabular}

${ }^{*}$ There were no statistically significant differences based on sex or time since treatment in the reporting of no symptoms versus any symptoms (some/moderate/yes combined).

†Remaining participants responded 'No'.

reported for the general population (median global health status 83 compared with 75 in the general population). ${ }^{8}$ Further, patients in our study rated their QoL as high despite substantial burden of fatigue. The observed QoL inflation may be due to altered expectations in the context of having faced life-threatening disease or patient selection. ${ }^{33}$

Musculoskeletal symptoms were the most common symptoms in our study. In clinical trials and observational studies of CIs, rates of musculoskeletal problems like arthralgia, myalgia and back pain have ranged widely, with rates of arthralgia as high as $43 \% .{ }^{34}{ }^{35}$ While clinical trials of CIs may underestimate rates of musculoskeletal irAEs, ${ }^{36}$ our study suggests that persistent musculoskeletal symptoms are moderately common in long-term survivors. While these symptoms were seldom reported as 'severe' on the PRO-CTCAE, the proportion of patients reporting at least some mobility problems on the EQ-5D-3L seemed to rise as patients survived longer after treatment (table 6). Further, while sex differences were not significant, we saw

Table 7 Immune-related adverse events

$$
\text { N (\%) }
$$

\begin{tabular}{lccc} 
Toxicity & Total $(\mathbf{n = 9 0 )}$ & Male $(\mathbf{n = 5 1 )}$ & $\begin{array}{l}\text { Female } \\
(\mathbf{n}=39)\end{array}$ \\
\hline Skin/rash & $34(37.8)$ & $18(35.3)$ & $16(41.0)$ \\
Endocrine & $24(26.7)$ & $12(23.5)$ & $12(31.0)$ \\
\hline Hypothyroidism & $18(20.0)$ & $8(15.7)$ & $10(25.6)$ \\
Hypophysitis & $10(11.1)$ & $5(9.8)$ & $5(12.8)$ \\
Colitis & $24(26.7)$ & $16(31.4)$ & $8(21.0)$ \\
Hepatitis & $15(16.7)$ & $10(20.0)$ & $5(12.8)$ \\
\hline Pulmonary & $8(8.9)$ & $4(7.8)$ & $4(10.3)$ \\
\hline Neuro & $7(7.8)$ & $6(11.8)$ & $1(2.6)$ \\
Musculoskeletal & $6(6.7)$ & $1(2.0)$ & $5(12.8)$ \\
Cardiac & $1(1.1)$ & $0(0.0)$ & $1(2.6)$ \\
Renal & $1(1.1)$ & $1(2.0)$ & $0(0.0)$ \\
Other & $13(14.4)$ & $6(11.8)$ & $7(18.0)$ \\
\hline
\end{tabular}

a trend toward higher rates of musculoskeletal symptoms in women than in men. Several studies have documented musculoskeletal problems in patients treated with CIs, ${ }^{35}$ though gender differences have not been described. As this group of survivors grows and patients live longer, future studies should further characterize gender-based differences and explore the evolution of musculoskeletal symptoms.

Fewer than half of patients in our sample $(41 \%)$ reported having seen a PCP in the last year; we did not evaluate whether these represented new versus established relationships. The Institute of Medicine recommends collaboration between oncologists and PCPs to optimize care for cancer survivors, ${ }^{37}$ and survivors' involvement with both an oncologist and a PCP is associated with higher quality care. ${ }^{38}$ PCPs, however, may lack adequate knowledge to deliver needed care ${ }^{39}$ Future studies should evaluate the trajectory of patient relationships with PCPs. As the population of CI-treated cancer survivors grows, there will be a need to ensure patient access to primary care and to educate PCPs about potential long-term health effects of CI therapy.

Our study has important limitations. Our sample size is relatively small, and our response rate was modest, so our findings may imperfectly reflect the experiences of the broader patient population. Further, due to the small sample size, we were unable to apply multivariable models to examine the observed associations adjusted for potential confounding factors. Our patients were also healthy prior to CI therapy and approved by their oncologist to be approached, which may have biased our sample toward healthier and more satisfied patients. Second, participants in our study received various CI regimens, often more than one, and duration of therapy varied (with median 15 months). Symptoms and QoL are likely to differ based on the drug received and the duration of therapy, but our study was not powered to detect differences based on these factors and the cross-sectional nature of our study does not enable understanding of changes in symptoms and QoL over time. Further, we collected only basic information on utilization of other health services; future 
prospective studies should describe the specific services needed in this population. In addition, we surveyed only patients who had survived for at least 1 year since initiation of CI therapy so our findings reflect this specific group; experiences of patients who respond less well to treatment may differ substantially. Finally, all participants in our study were patients at a single institution and therefore may not be representative of metastatic melanoma patients across the nation. Long-term health outcomes data, including information on symptom burden and QoL, are needed from prospective studies of larger and more diverse cohorts of patients treated with CI therapy, which will likely come from studies other than those focused on melanoma.

In conclusion, our cross-sectional study found that patients with advanced melanoma who were off treatment and had survived at least 1 year after therapy with CI had good QoL. Skin and musculoskeletal symptoms, moderate problems with anxiety and depression, and fatigue were moderately prevalent but appeared not to severely impede function or QoL. Our findings are reassuring that this emerging population of cancer survivors can live not only longer but better than in years past.

\section{Twitter Jessica A Lavery @jessicalavs and Deborah Korenstein @DKorenstein}

Acknowledgements The authors wish to thank Cheryl Beebe and James Grimm for support of this research.

Contributors DK and MM had full access to all the data in the study and take responsibilitiy for the integrity of the data and the accuracy of the data analysis. SSB and DK designed the study. MM, JAL, RS, BT, NK and DK acquired, analyzed or interepreted the data. MM and DK drafted the manuscript; JAL and MM performed statistical analysis; SSB obtained funding; MM provided administrative, technical or material support; DK supervized the study. All authors provided critical revisions, gave final approval and agreed to be accountable for the work.

Funding This work was supported in part by the Beebe/Grimm Fund and by a Cancer Center Support Grant from the National Cancer Institute to Memorial Sloan Kettering Cancer Centre (P30 CA008748 to MP, SSB, JJM, RS, JW and DK). JW's work was also supported in part by Ludwig Collaborative and Swim Across America Laboratory, and Parker Institute for Cancer Immunotherapy.

Competing interests SSB is an employee of Flatiron Health which is a subsidiary of Roche. JW is a consultant for: Adaptive Biotech; Advaxis; Amgen; Apricity; Array BioPharma; Ascentage Pharma; Astellas; Bayer; Beigene; Bristol Myers Squibb; Celgene; Chugai; Elucida; Eli Lilly; F Star; Genentech; Imvaq; Janssen; Kleo Pharma; Kyowa Hakko Kirin; Linneaus; Medlmmune; Merck; Neon Therapuetics; Northern Biologics; Ono; Polaris Pharma; Polynoma; Psioxus; Puretech; Recepta; Takara Bio; Trieza; Sellas Life Sciences; Serametrix; Surface Oncology; Syndax; Syntalogic. Research support: Bristol Myers Squibb; Medimmune; Merck Pharmaceuticals; Genentech. Equity in: Potenza Therapeutics; Tizona Pharmaceuticals; Adaptive Biotechnologies; Elucida; Imvaq; Beigene; Trieza; Linneaus; Honoraium: EsanexMP is a consultant for: BMS; Merck; Array BioPharma; Novartis; Aduro; Incyte; NewLink Genetics. Institutional support for: BMS; Merck; RGenix; Infinity; AstraZeneca; Novartis; Array BioPharma. Honoraria: BMS and Merck. DK's spouse serves on the scientific advisory board of Vedanta Biosciences and is a consultant for Takeda. JAL discloses salary support for Project Genomics Evidence Neoplasia Information Exchange (GENIE) through the American Association for Cancer Research.

\section{Patient consent for publication Not required.}

Ethics approval This study was conducted in compliance with the Declaration of Helsinki, and in accordance with local legal and regulatory requirements. The protocol was approved by MSK's Institutional Review Board under IRB 17-518.

Provenance and peer review Not commissioned; externally peer reviewed.

Data availability statement No data are available. The datasets generated and/ or analyzed during the current study are not publicly available due to patient confidentiality reasons but are available from the corresponding author on reasonable request.

Open access This is an open access article distributed in accordance with the Creative Commons Attribution Non Commercial (CC BY-NC 4.0) license, which permits others to distribute, remix, adapt, build upon this work non-commercially, and license their derivative works on different terms, provided the original work is properly cited, appropriate credit is given, any changes made indicated, and the use is non-commercial. See http://creativecommons.org/licenses/by-nc/4.0/.

\section{ORCID iD}

Deborah Korenstein http://orcid.org/0000-0003-1859-0574

\section{REFERENCES}

1 Hodi FS, Chiarion-Sileni V, Gonzalez R, et al. Nivolumab plus ipilimumab or nivolumab alone versus ipilimumab alone in advanced melanoma (CheckMate 067): 4-year outcomes of a multicentre, randomised, phase 3 trial. Lancet Oncol 2018;19:1480-92.

2 Johnson DB, Friedman DL, Berry E, et al. Survivorship in immune therapy: assessing chronic immune toxicities, health outcomes, and functional status among long-term ipilimumab survivors at a single referral center. Cancer Immunol Res 2015;3:464-9.

3 Schadendorf D, Larkin J, Wolchok J, et al. Health-Related quality of life results from the phase III CheckMate 067 study. Eur J Cancer 2017;82:80-91.

4 Ford I, Norrie J. Pragmatic trials. N Engl J Med 2016;375:454-63.

5 Langbaum T, Smith TJ. Time to study Metastatic-Cancer survivorship. N Engl J Med 2019;380:1300-2.

6 Harris PA, Taylor R, Thielke R, Payne J, et al. Research electronic data capture (REDCap)--a metadata-driven methodology and workflow process for providing translational research informatics support. J Biomed Inform 2009;42:377-81.

7 Harris RT PA, Minor BL, Elliott V, et al. REDCap Consortium, the REDCap Consortium: building an international community of software partners. J Biomed Inform 2019.

8 Aaronson NK, Ahmedzai S, Bergman B, et al. The European organization for research and treatment of cancer QLQ-C30: a quality-of-life instrument for use in international clinical trials in oncology. J Natl Cancer Inst 1993;85:365-76.

9 EuroQol Research Foundation. EQ-5D-3L user guide.

10 . Available: https://healthcaredelivery.cancer.gov/pro-ctcae/

11 Krupp LB, LaRocca NG, Muir-Nash J, et al. The fatigue severity scale. Application to patients with multiple sclerosis and systemic lupus erythematosus. Arch Neurol 1989;46:1121-3.

12 de Souza JA, Yap BJ, Wroblewski K, et al. Measuring financial toxicity as a clinically relevant patient-reported outcome: the validation of the comprehensive score for financial toxicity (cost). Cancer 2017;123:476-84.

13 StataCorp. Stata statistical software: release 15. College Station TSL, 2017.

14 Kaur H, Bollu PC. Chronic Insomnia. In: StatPearls. Treasure Island (FL): StatPearls Publishing StatPearls Publishing LLC, 2019.

15 Behringer K, Goergen $\mathrm{H}$, Müller $\mathrm{H}$, et al. Cancer-Related fatigue in patients with and survivors of Hodgkin lymphoma: the impact on treatment outcome and social reintegration. J Clin Oncol 2016;34:4329-37.

16 Cella D, Davis K, Breitbart W, et al. Cancer-Related fatigue: prevalence of proposed diagnostic criteria in a United States sample of cancer survivors. J Clin Oncol 2001;19:3385-91.

17 Matias M, Baciarello G, Neji M, et al. Fatigue and physical activity in cancer survivors: a cross-sectional population-based study. Cancer Med 2019;8:2535-44.

18 Leysen L, Lahousse A, Nijs J, et al. Prevalence and risk factors of sleep disturbances in breast cancersurvivors: systematic review and meta-analyses. Support Care Cancer 2019;27:4401-33.

19 Abdel-Rahman O, Helbling D, Schmidt J, et al. Treatment-associated fatigue in cancer patients treated with immune checkpoint inhibitors; a systematic review and meta-analysis. Clin Oncol 2016;28:e127-38.

20 Lai-Kwon J, Khoo C, Lo S, et al. The survivorship experience for patients with metastatic melanoma on immune checkpoint and BRAF-MEK inhibitors. J Cancer Surviv 2019;13:503-11.

21 Tibubos AN, Ernst M, Brähler E, et al. Fatigue in survivors of malignant melanoma and its determinants: a register-based cohort study. Support Care Cancer 2019;27:2809-18.

22 Singer S, Kuhnt S, Zwerenz R, et al. Age- and sex-standardised prevalence rates of fatigue in a large hospital-based sample of cancer patients. Br J Cancer 2011;105:445-51. 
23 Goedendorp MM, Gielissen MFM, Verhagen CAHHVM, et al. Development of fatigue in cancer survivors: a prospective follow-up study from diagnosis into the year after treatment. J Pain Symptom Manage 2013;45:213-22.

24 Park W, Lee J-K, Kim C-R, et al. Factors associated with fatigue in Korean gastric cancer survivors. Korean $\mathrm{J}$ Fam Med 2015;36:328-34.

25 Bower JE, Bak K, Berger A, et al. Screening, assessment, and management of fatigue in adult survivors of cancer: an American Society of clinical oncology clinical practice guideline adaptation. $J$ Clin Oncol 2014;32:1840-50.

26 Revicki DA, van den Eertwegh AJM, Lorigan P, et al. Health related quality of life outcomes for unresectable stage III or IV melanoma patients receiving ipilimumab treatment. Health Qual Life Outcomes 2012;10:66.

27 Coens C, Suciu S, Chiarion-Sileni V, et al. Health-related quality of life with adjuvant ipilimumab versus placebo after complete resection of high-risk stage III melanoma (EORTC 18071): secondary outcomes of a multinational, randomised, double-blind, phase 3 trial. Lancet Oncol 2017;18:393-403.

28 Beutel ME, Fischbeck S, Binder H, et al. Depression, anxiety and quality of life in long-term survivors of malignant melanoma: a register-based cohort study. PLoS One 2015;10:e0116440.

29 Kent EE, Ambs A, Mitchell SA, et al. Health-Related quality of life in older adult survivors of selected cancers: data from the SEER-MHOS linkage. Cancer 2015;121:758-65.

30 Weaver KE, Forsythe LP, Reeve BB, et al. Mental and physical health-related quality of life among U.S. cancer survivors: population estimates from the 2010 National health interview survey. Cancer Epidemiol Biomarkers Prev 2012;21:2108-17.

31 Lin VW, Blaylock B, Epstein J, et al. Systematic literature review of health-related quality of life among aggressive non-Hodgkin lymphoma survivors. Curr Med Res Opin 2018;34:1529-35.

32 Doege D, Thong MS-Y, Koch-Gallenkamp L, et al. Health-Related quality of life in long-term disease-free breast cancer survivors versus female population controls in Germany. Breast Cancer Res Treat 2019;175:499-510.

33 Addington-Hall J, Kalra L. Who should measure quality of life? BMJ 2001;322:1417-20.

34 Baxi S, Yang A, Gennarelli RL, et al. Immune-Related adverse events for anti-PD-1 and anti-PD-L1 drugs: systematic review and metaanalysis. BMJ 2018;360:k793.

35 Cappelli LC, Gutierrez AK, Bingham CO, et al. Rheumatic and musculoskeletal immune-related adverse events due to immune checkpoint inhibitors: a systematic review of the literature. Arthritis Care Res 2017;69:1751-63.

36 Yang A, Baxi S, Korenstein D. Clinicaltrials. Gov for facilitating rapid understanding of potential harms of new drugs: the case of checkpoint inhibitors. JOP 2018;14:72-6.

37 Institute of Medicine and National Research Council. From cancer patient to cancer survivor: lost in transition. Washington, 2006.

38 Earle CC, Neville BA. Under use of necessary care among cancer survivors. Cancer 2004;101:1712-9.

39 Lawrence RA, McLoone JK, Wakefield CE, et al. Primary care physicians' perspectives of their role in cancer care: a systematic review. J Gen Intern Med 2016;31:1222-36. 
Correction: Quality of life in long-term survivors of advanced melanoma treated with checkpoint inhibitors

Mamoor M, Postow MA, Lavery JA, et al. Quality of life in long-term survivors of advanced melanoma treated with checkpoint inhibitors. JImmunother Cancer 2020;8:e000260. doi: 10.1136/jitc-2019-000260.

Since the online publication of this article, the authors have noticed that the middle initial for author Jedd Wolchok is incorrect. The correct spelling is Jedd D Wolchok.

(C) Author(s) (or their employer(s)) 2020. No commercial re-use. See rights and permissions. Published by BMJ.

J Immunother Cancer 2020;8:e000260corr1. doi:10.1136/jitc-2019-000260corr1

D Check for updates 